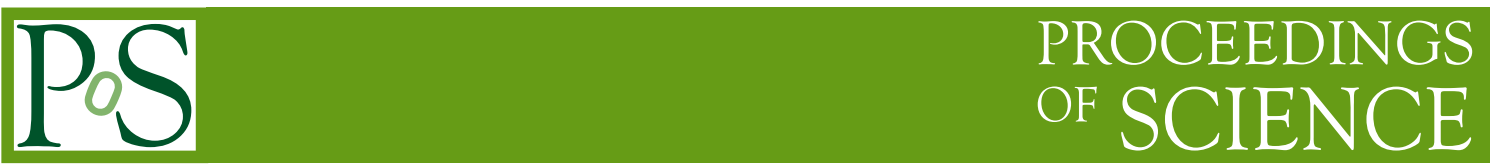

\title{
Simulation of Irradiated Si Detectors
}

\author{
Ranjeet Dalal*, Ashutosh Bhardwaj, Kirti Ranjan, Kavita Lalwani and Geetika Jain \\ CDRST, Department of physics and Astrophysics, University of Delhi, India \\ E-mail: rdalalecern.ch
}

The expected increments in the radiation fluences to which the Si sensors will be exposed after future upgrades of LHC demands the systematic investigation of radiation damage of silicon sensors. The campaigns to produce radiation hard Si sensors have already been initiated by CMS and ATLAS. The experimental investigation of radiation damage should be complemented by simulations of silicon sensors with proper radiation damage modeling. The radiation damage modeling not only provides insight in the understanding of the radiation damage but, it is also helpful in the sensor design optimization. The radiation damage simulation of silicon sensors are needed to be carried out by simultaneous incorporation of appropriate bulk and surface damages since both the strip and pixel sensors undergo these degrading effects. The use of either bulk damage or surface damage alone can lead to wrong conclusions. In this work, simulation of irradiated silicon sensors incorporating the bulk and surface damages, using TCAD tool (Silvaco), are discussed. The bulk damage is parametrized by two trap model while the surface damage is incorporated in the simulations using oxide charge density $\left(\mathrm{Q}_{\mathrm{F}}\right)$ and interface trap density $\left(\mathrm{N}_{\mathrm{it}}\right)$.

The 23rd International Workshop on Vertex Detectors,

15-19 September 2014

Macha Lake, The Czech Republic

${ }^{*}$ Speaker. 


\section{Introduction}

Silicon sensors in strip and pixel configurations have been widely used in the present and past high energy physics (HEP) experiments $[1,2,3]$. These sensors have shown excellent operational performance in the radiation environments of present trackers of the LHC experiments [2,3]. These sensors are expected to prevail their dominance in the trackers of High Luminosity LHC (HL-LHC) era $[4,5]$ as well. Since the silicon sensors are used near the collision point in the high energy experiments, they are irradiated with very high particle fluence. For example, during LHC run after the Phase-II upgrade, the CMS strip sensors may face fluence as high as $1.10^{15} \mathrm{n}_{\mathrm{eq}} \mathrm{cm}^{-2}\left(\mathrm{n}_{\mathrm{eq}}\right.$ stands for $1 \mathrm{MeV}$ neutron equivalent fluence following the NIEL (Non Ionizing Energy Loss) hypothesis and the expected fluence for the inner layers of pixel is of the order of $\approx 1.10^{16} \mathrm{n}_{\mathrm{eq}} \mathrm{cm}^{-2}$. The present sensor designs are not capable to withstand such harsh radiation fluences [4] and systematic radiation hardness investigation for silicon sensors for the CMS and the ATLAS detectors are ongoing $[4,5]$.

During the sensor operation in HEP experiments, high irradiation fluence can cause deformations in silicon crystal and can degrade the $\mathrm{SiO}_{2}$ properties as well, resulting in both the bulk and surface damages in silicon sensors. The bulk damage can introduce acceptor and donor type traps inside the band gap of silicon. These traps can act as recombination/generation centers and trapping centers for the charge carriers, thus increasing the leakage current, decreasing the charge collection efficiencies (CCE) and changing the space charge inside the irradiated silicon sensor [6]. On the other hand, surface damage leads to higher positive oxide charge density $\left(\mathrm{Q}_{\mathrm{F}}\right)$ and higher interface trap density $\left(\mathrm{N}_{\mathrm{it}}\right)[7,8]$. The positive charge layer due to $\mathrm{Q}_{\mathrm{F}}$ leads to formation of electron accumulation layer below interface, thus disrupting the inter strip resistance $\left(\mathrm{R}_{\text {int }}\right)$ for $\mathrm{n}$-on-p strip sensors. To counter the effect of $\mathrm{Q}_{\mathrm{F}}$, p-stop and p-spray strip insulation methods [9] are commonly used between $\mathrm{n}^{+}$strips. Moreover, the interface traps can also play a significant role in deciding the surface properties of sensors as significant fraction of these traps are deep traps and their density is comparable to $\mathrm{Q}_{\mathrm{F}}[8]$.

Many excellent radiation damage simulation works for the silicon sensors have been reported in the past. Some of these works are listed in references $[9,10,11,12,13]$. These simulations either reported the investigation of the effects of $\mathrm{Q}_{\mathrm{F}}$ variation on the surface properties of sensors or considered the linear bulk trap variations with fluence, using approximate trap models to mimic the effect of the bulk radiation damage. However, real strip and pixel silicon sensors used in the hadron environment suffer both the surface and bulk damage simultaneously. Deep inside the bulk, only bulk damage is important, but near the surface, effect of space charges due to bulk and surface damage are inter-woven. Conclusions drawn from the simulations which are implementing either surface damage or bulk damage alone may have limited applicability. Moreover, surface damage has been implemented in the past TCAD simulations using the variation of $\mathrm{Q}_{\mathrm{F}}$ only. But it is well known [8] that interface trap density generated because of dangling silicon bonds is an equally important component of surface damage. The density of these interface trap states $\left(\mathrm{N}_{\mathrm{it}}\right)$ increases many fold with irradiation and saturates around 2-3 $\cdot 10^{12} \mathrm{~cm}^{-2}$. A significant fraction of these interface trap states are deep traps inside the band gap of silicon and thus they can influence the interface properties of strip and pixel sensors.

In this work, simultaneous implementation of surface and bulk damage is carried out into TCAD 
(Silvaco) [14] simulations. Bulk damage is approximated by two trap model, a donor trap and an acceptor trap. Surface damage is implemented using the measured trends of $\mathrm{Q}_{\mathrm{F}}$ and $\mathrm{N}_{\mathrm{it}}[7,8]$. Silvaco TCAD solves Poisson's equation and continuity equation for the charge carriers. The default parameters were used for the concentration dependent lifetime, Shockley-Read-Hall (SRH) recombination and for concentration and field dependent mobility models. The Selberherr impact ionization model is included in the simulation. The trap assisted tunnelling is also used to include the change in effective lifetime of trapped carriers at high values of electric field within the sensor. Calculations are performed using a triangular grid generated by the DevEdit (Silvaco). Reflecting Neumann conditions are imposed at the outer edges of the structure and also on the top of $\mathrm{SiO}_{2}$.

The TCAD simulations for $n-o n-p$ and $p$-on- $n$ strip sensors are performed using three strip simulation structure with strip pitch of $80 \mu \mathrm{m}$, strip width of $18 \mu \mathrm{m}$ and thickness of $320 \mu \mathrm{m}$. Si substrate with orientation $<100>$ having a uniform doping concentration of $3.10^{12} \mathrm{~cm}^{-3}$ is used. The strip implants have a Gaussian profile with a peak concentration of $5.10^{18} \mathrm{~cm}^{-3}$ and a junction depth equal to $2 \mu \mathrm{m}$. The $\mathrm{SiO}_{2}$ thickness below the metal overhang and between the electrodes is 700 $\mathrm{nm}$ and $1 \mu \mathrm{m}$ respectively. For n-on-p strip sensors, a double p-stop structure is used between the $\mathrm{n}^{+}$strip implants. The width of each p-stop is $4 \mu \mathrm{m}$ with a depth of $1.5 \mu \mathrm{m}$ and they are separated by $6 \mu \mathrm{m}$. It is assumed that the lateral spread for the implantations is equal to 0.7 times the vertical depth. The backside contact is implemented using an implant depth (where backside implant concentration becomes same as that of bulk concentration) of $30 \mu \mathrm{m}$. For the $\mathrm{R}_{\text {int }}$ simulations, a small $0.2 \mathrm{~V}$ bias is given to the central electrodes, keeping the neighboring electrodes shorted while reverse bias is provided from the backside electrode. $\mathrm{R}_{\text {int }}$ is calculated from the difference in central and neighboring electrode currents. $R_{\text {int }}$ values are normalized for strip length equal to $1 \mathrm{~cm}$. Further details about simulation structure are given in [15].

\section{Radiation damage implementation in TCAD}

\subsection{Bulk damage}

In TCAD simulations for irradiated sensors, it is very difficult to consider actual trap levels inside the silicon band gap, partly because of the uncertainty in various trap related parameter measurements and partly due to cluster defects, which are not well understood [6]. Hence, an approximate bulk damage model incorporating just two trap levels, one acceptor and one donor, is devised for proton irradiated sensors. The resulting bulk damage model is able to account for the various macroscopic measured properties of proton irradiated silicon sensors like leakage current, full depletion voltage and decrease in charge collection efficiency with increase in fluence. Various parameters of bulk damage model are listed in Table 1. The trap density for a given fluence $\left(\mathrm{n}_{\mathrm{eq}} \mathrm{cm}^{-2}\right)$ is calculated using the parameter named introduction rate such that trap density $=$ Introduction rate $\times$ fluence.

A comparison of the simulated ( $1 \mu \mathrm{m} \times 1 \mu \mathrm{m} \times 300 \mu \mathrm{m}$ silicon pad diode) and expected leakage currents for various fluence values at $253 \mathrm{~K}$ is shown in figure 1a. The expected leakage current is calculated using,

$$
I=\alpha \Phi_{\mathrm{eq}} V
$$


Table 1: List of parameters for the two trap bulk damage model (Model 1) used in the simulations. $\sigma_{\mathrm{e}}$ and $\sigma_{\mathrm{h}}$ represent the electron and hole capture cross-sections of the trap respectively.

\begin{tabular}{|c|c|c|c|c|}
\hline Trap Type & $\begin{array}{c}\text { Energy Level } \\
(\mathrm{eV})\end{array}$ & $\begin{array}{c}\text { Introduction Rate } \\
\left(\mathrm{cm}^{-1}\right)\end{array}$ & $\begin{array}{c}\sigma_{\mathrm{e}} \\
\left(\mathrm{cm}^{2}\right)\end{array}$ & $\begin{array}{c}\sigma_{\mathrm{h}} \\
\left(\mathrm{cm}^{2}\right)\end{array}$ \\
\hline Acceptor & $\mathrm{E}_{C}-0.51 \mathrm{eV}$ & 4 & $2.10^{-14}$ & $2.6 .10^{-14}$ \\
\hline Donor & $\mathrm{E}_{V}+0.48 \mathrm{eV}$ & 3 & $2.10^{-14}$ & $2.10^{-14}$ \\
\hline
\end{tabular}

Where $\alpha$ is damage coefficient [17] $\left(8.8 \cdot 10^{-19} \mathrm{~cm}^{-1}\right.$ at $\left.253 \mathrm{~K}\right), \Phi_{\mathrm{eq}}$ is $1 \mathrm{MeV}$ neutron equivalent fluence and $\mathrm{V}$ is sensor volume. Further, detailed full depletion voltage $\left(\mathrm{V}_{\mathrm{FD}}\right)$ simulations are discussed in section 3.1, where a good agreement is achieved between the simulated and measured $\mathrm{V}_{\mathrm{FD}}$ for irradiated silicon sensors.

Though the above approximate two trap bulk damage model can account for the leakage current
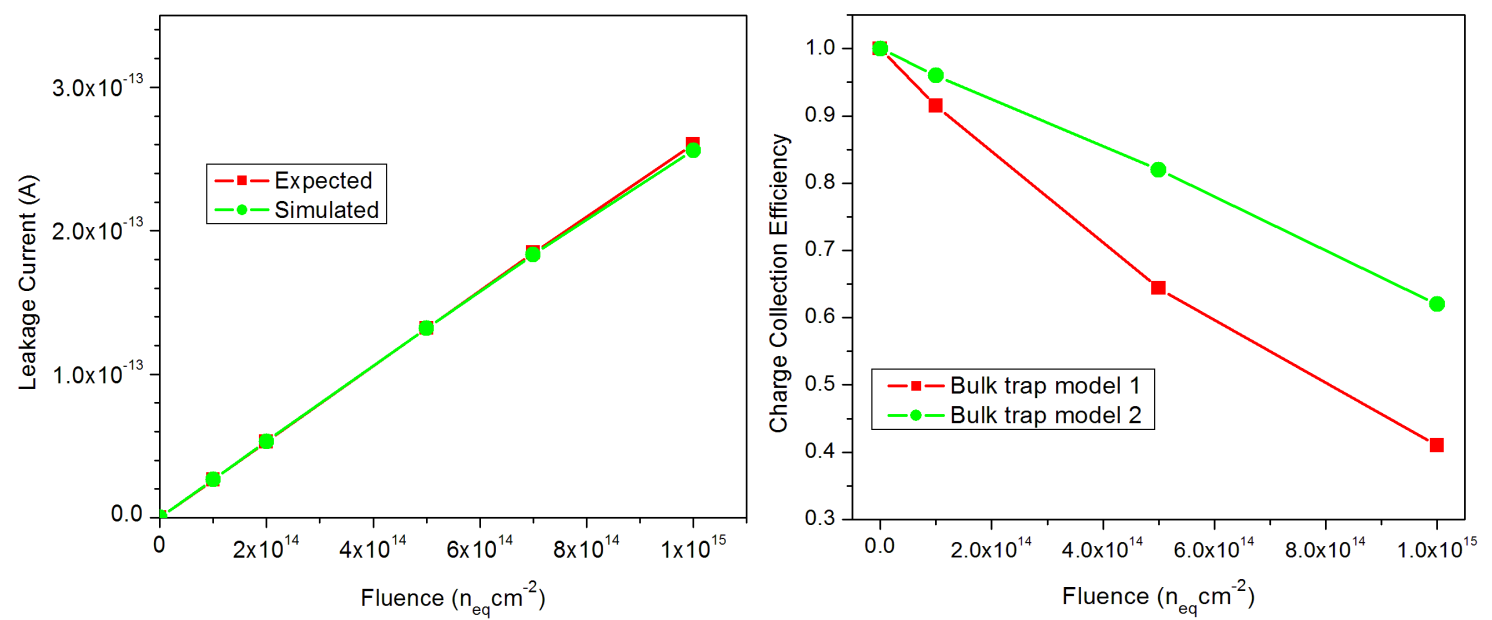

Figure 1: a.) Simulated and expected leakage current variation with fluence at $253 \mathrm{~K}$ b.) simulated CCE variation with fluence for bulk damage model listed in table 1 (Model 1) and for Model 2 (described in text below) at $253 \mathrm{~K}$. CCE simulations are carried out for infra red laser of frequency $1060 \mathrm{~nm}$. Damage coefficient [17] $\alpha=8.8 \cdot 10^{-19} \mathrm{~cm}^{-1}$ at $253 \mathrm{~K}$ is used to evaluate the expected value of leakage current.

and $\mathrm{V}_{\mathrm{FD}}$ of proton irradiated diode, the bulk damage model formulation is not unique. One can devise a range of bulk damage models [13] capable of producing similar trends for current, $\mathrm{V}_{\mathrm{FD}}$ and electric field (double junction for proton irradiated silicon sensors). In Silvaco TCAD, one of the possible way of formulating equivalent trap models capable of producing similar leakage currents and $\mathrm{V}_{\mathrm{FD}}$ values is to reduce the capture cross sections of donor trap and simultaneous increase of capture cross sections for acceptor trap (particularly $\sigma_{\mathrm{h}}$ ). These trap models may have different charge collection efficiencies due to different amount of charge carrier trapping. An example is shown in figure $1 \mathrm{~b}$, where CCE comparison is shown for Model 1 (whose parameters are listed in table 1) and Model 2 (whose parameters are listed in table 2). For Model 2, the e/h capture cross sections for donor trap levels are decreased but hole capture cross section for acceptor trap level is increased compared to Model 1. These two bulk damage models produce quite similar leakage 
current and $\mathrm{V}_{\mathrm{FD}}$ values but different $\mathrm{CCE}$ at higher fluences. In this work, all other simulations are carried out using the bulk damage model listed in table 1.

Table 2: List of parameters for Model 2.

\begin{tabular}{|c|c|c|c|c|}
\hline Trap Type & $\begin{array}{c}\text { Energy Level } \\
(\mathrm{eV})\end{array}$ & $\begin{array}{c}\text { Introduction Rate } \\
\left(\mathrm{cm}^{-1}\right)\end{array}$ & $\begin{array}{c}\sigma_{\mathrm{e}} \\
\left(\mathrm{cm}^{2}\right)\end{array}$ & $\begin{array}{c}\sigma_{\mathrm{h}} \\
\left(\mathrm{cm}^{2}\right)\end{array}$ \\
\hline Acceptor & $\mathrm{E}_{C}-0.51 \mathrm{eV}$ & 4 & $2.10^{-14}$ & $3.8 .10^{-14}$ \\
\hline Donor & $\mathrm{E}_{V}+0.48 \mathrm{eV}$ & 3 & $2.10^{-15}$ & $2.10^{-15}$ \\
\hline
\end{tabular}

\subsection{Surface damage}

The effect of surface damage in device simulations is incorporated by using different values of $\mathrm{Q}_{\mathrm{F}}$ and $\mathrm{N}_{\mathrm{it}}$ at the $\mathrm{Si}-\mathrm{SiO}_{2}$ interface. The $\mathrm{Q}_{\mathrm{F}}$ and $\mathrm{N}_{\mathrm{it}}$ values used in the present work are chosen in accordance with the measurements [7, 8].

\subsubsection{Oxide charge density $\left(Q_{F}\right)$}

Along with bulk damage in silicon crystal, proton irradiation can also create electron-hole (e/h) pairs in the $\mathrm{SiO}_{2}$. Though most of these e/h pairs recombine, but the remaining electrons and holes can move in $\mathrm{SiO}_{2}$ by drift and diffusion mechanisms, escaping the initial recombination [8]. Since electron mobility in $\mathrm{SiO}_{2}\left(\approx 20 \mathrm{~cm}^{2} / \mathrm{Vs}\right)$ is much higher than the hole mobility in $\mathrm{SiO}_{2}$ (which is less than $10^{-5} \mathrm{~cm}^{2} / \mathrm{Vs}$ ), a significant fraction of free electrons escape from $\mathrm{SiO}_{2}$, leaving behind holes trapped near $\mathrm{Si}_{-} \mathrm{SiO}_{2}$ interface, forming a positive charge layer. This positive charge layer is termed as oxide charge density or interface charge density $\left(\mathrm{Q}_{\mathrm{F}}\right)$.

Extensive measurements of $\mathrm{Q}_{\mathrm{F}}$ and $\mathrm{N}_{\mathrm{it}}$ for different ionization doses (in Gray) of $\mathrm{X}$-ray have been reported in ref. [7, 8]. For proton irradiated sensors, the estimation of the total ionization dose (TID) can be carried out using the stopping power $(d E / d x)$ for protons in $\mathrm{SiO}_{2}$ [18]. The calculated TID equivalent of proton fluence can be used to inferre the $\mathrm{Q}_{\mathrm{F}}$ and $\mathrm{N}_{\mathrm{it}}$ from measurements [8]. For proton fluence of $1.10^{15} \mathrm{n}_{\mathrm{eq}} \mathrm{cm}^{-2}$ minimum TID value (which can be calculated using minimum $d E / d X$ for protons [18]) is about $0.44 \mathrm{MGy}$.

Since $Q_{F}$ value increases with the total ionization dose (TID) and saturates around $2.10^{12} \mathrm{~cm}^{-2}$ for X-ray doses of about $1 \mathrm{MGy}$, we have implemented the $\mathrm{Q}_{\mathrm{F}}$ value of about $1-2.10^{12} \mathrm{~cm}^{-2}$ into TCAD simulations for the proton fluence of $1.10^{15} \mathrm{n}_{\mathrm{eq}} \mathrm{cm}^{-2}$. For lower fluences, lower $\mathrm{Q}_{\mathrm{F}}$ values are implemented keeping $\mathrm{Q}_{\mathrm{F}}$ measurements in view. It may be noted that $\mathrm{Q}_{\mathrm{F}}$ values are complex function of TID rate, fabrication process, applied electric field, wafer quality and measurement conditions (humidity etc.). Hence, range of $\mathrm{Q}_{\mathrm{F}}$ is used for simulations at the given fluence value. In present work, $\mathrm{Q}_{\mathrm{F}}$ is implemented as a positive charge-sheet located at the $\mathrm{Si}_{-} \mathrm{SiO}_{2}$ interface with a uniform distribution along the interface.

\subsubsection{Interface trap density $\left(\mathrm{N}_{\mathrm{it}}\right)$}

Along with $\mathrm{Q}_{\mathrm{F}}$ increments, ionizing radiation can also increase the interface trap density [8]. Interface traps can play an important role in deciding the surface properties of silicon sensors because of the two main reasons. First, the density of the interface states is comparable to the oxide 
charge density [8]. Second, a significant number of these interface states are deep trap states, thus these are capable of altering the space charge near interface. The prominent trap peak in the measured thermal dielectric relaxation current (TDRC) spectra for MOS (Metal-Oxide-Semiconductor) structures is attributed to th $0.6 \mathrm{eV}$ deep trap.

There is ambiguity about the nature of $\mathrm{N}_{\mathrm{it}}$ in TDRC measurements, but $\mathrm{R}_{\mathrm{int}}$ simulations and their comparisons with the measured X-ray irradiated strips sensors indicate that most of the interface trap states should be acceptor type [16]. In this work, we have assumed that for a given interface traps density, $60 \%$ of the $\mathrm{N}_{\mathrm{it}}$ are deep acceptor traps $\left(\mathrm{E}_{C}-0.60 \mathrm{eV}\right)$ and $40 \%$ of $\mathrm{N}_{\mathrm{it}}$ are shallow acceptor traps $\left(E_{C}-0.39 \mathrm{eV}\right)$ with e/h capture cross sections equal to $1.10^{-15} \mathrm{~cm}^{2}$. Since the measured $\mathrm{Q}_{\mathrm{F}}$ and $\mathrm{N}_{\mathrm{it}}$ values [8] are quite similar in magnitude, we have implemented the $\mathrm{N}_{\mathrm{it}}$ value equal to $\mathrm{Q}_{F}$ value in each simulation, unless otherwise stated.

It is interesting to analyze the separate and combined effects of $\mathrm{Q}_{\mathrm{F}}, \mathrm{N}_{\mathrm{it}}$ and bulk traps on the flatband voltage shift for MOS. Flatband voltage simulations for a p-type MOS having $\mathrm{SiO}_{2}$ layer of thickness equal to $670 \mathrm{~nm}$ along with a $\mathrm{Si}_{3} \mathrm{~N}_{4}$ layer of thickness $50 \mathrm{~nm}$ using AC frequency equal to $1 \mathrm{KHz}$ are shown in figure 2 . These simulations are carried out using a simple $1 \mu \mathrm{m} \times 1 \mu \mathrm{m} \times$ $320 \mu \mathrm{m}$ silicon structure with $30 \mu \mathrm{m}$ backside doping depth. The flatband voltage shift due to the $\mathrm{Q}_{\mathrm{F}}$ is opposite to that of $\mathrm{N}_{\mathrm{it}}$ as $\mathrm{Q}_{\mathrm{F}}$ results in a positive charge layer along the interface, while $\mathrm{N}_{\mathrm{it}}$ leads to negative space charge along the interface due to acceptor nature of the interface traps.

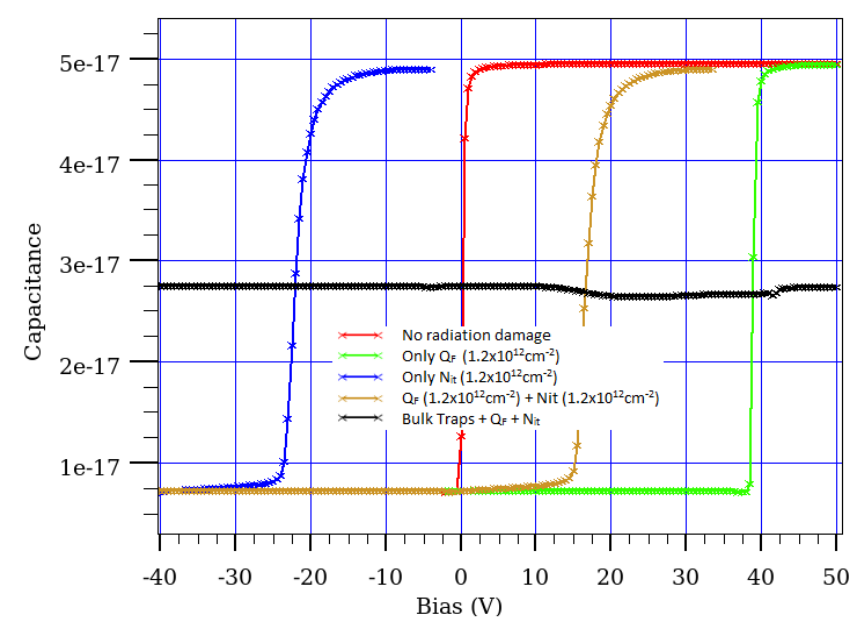

Figure 2: Simulated flatband voltages for $\mathrm{Q}_{\mathrm{F}}, \mathrm{N}_{\mathrm{it}}$ and bulk damage (for fluence $=1.10^{15} \mathrm{n}_{\mathrm{eq}} \mathrm{cm}^{-2}$ ) for p-type MOS at $293 \mathrm{~K}$.

\section{Simulation results}

\subsection{Effect of bulk traps on full depletion voltage $\left(\mathrm{V}_{\mathrm{FD}}\right)$}

The effect of bulk traps on the full depletion voltage was investigated using a simple diode structure of dimensions $1 \mu \mathrm{m}$ x $1 \mu \mathrm{m}$ x $300 \mu \mathrm{m}$. The $\mathrm{V}_{\mathrm{FD}}$ values are extracted from the $1 / C_{\mathrm{diff}}^{2}$ plots where $\mathrm{C}_{\text {diff }}$ (backplane capacitance) simulations for different reverse bias voltages are carried 
out at $\mathrm{AC}$ frequency of $1 \mathrm{KHz}$. To investigate the effect of initial bulk doping on $\mathrm{V}_{\mathrm{FD}}$, three different initial bulk doping values $2.10^{11} \mathrm{~cm}^{-3}, 2.10^{12} \mathrm{~cm}^{-3}$ and $4.10^{12} \mathrm{~cm}^{-3}$ are considered. Further, $\mathrm{V}_{\mathrm{FD}}$ simulations are carried out for both n-on-p and p-on-n diodes.

It can be deduced from figure 3 that for low fluence values, $V_{F D}$ decreases with the increase in
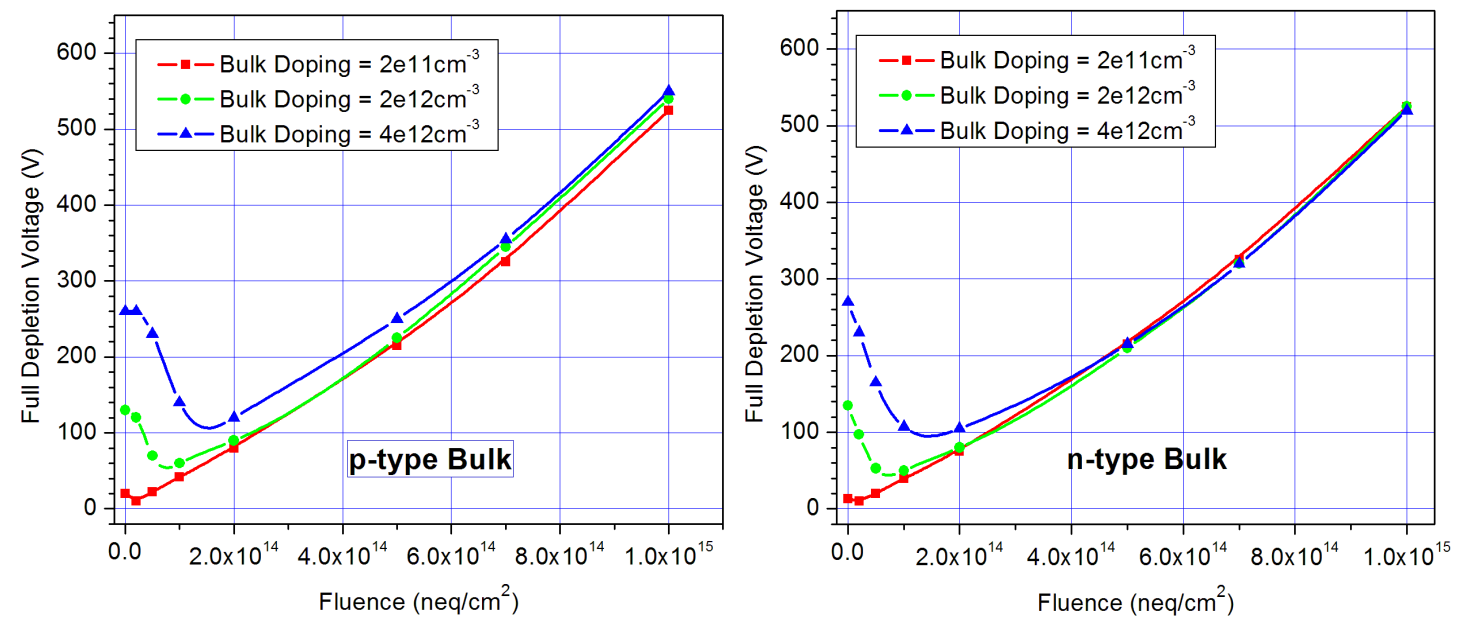

Figure 3: a.) Simulated $V_{B D}$ for n-on-p and , b.) for p-on-n diodes for different initial bulk doping at $253 \mathrm{~K}$.

the fluence, becomes minimum and then for higher fluences, $\mathrm{V}_{\mathrm{FD}}$ increases sharply. The $\mathrm{V}_{\mathrm{FD}}$ minimum for bulk doping $2 \cdot 10^{11} \mathrm{~cm}^{-3}$ happens at very low fluence $\left(\approx 2 \cdot 10^{13} \mathrm{n}_{\mathrm{eq}} \mathrm{cm}^{-2}\right)$ but for higher initial dopings, the $\mathrm{V}_{\mathrm{FD}}$ minimum takes place at higher fluences (say for initial doping 4 . $10^{12} \mathrm{~cm}^{-3}, \mathrm{~V}_{\mathrm{FD}}$ minimum is for fluence $\approx 1.5 \cdot 10^{14} \mathrm{n}_{\mathrm{eq}} \mathrm{cm}^{-2}$. For the fluence higher than $\approx$ 3. $10^{14} \mathrm{n}_{\mathrm{eq}} \mathrm{cm}^{-2}$, full depletion voltage is practically independent of initial dopings used in the simulations, which indicates the predominant role of bulk traps for higher fluences. Similar observations have been made in the measurements as well [19,20].

One is tempted to attribute the effect of initial $\mathrm{V}_{\mathrm{FD}}$ lowering with fluence to Donor Removal or Acceptor Removal, but in the $\mathrm{V}_{\mathrm{FD}}$ simulations in figure 3, initial bulk dopings are kept same. The initial $\mathrm{V}_{\mathrm{FD}}$ lowering is due to the trap effects only. For the non-irradiated sensor, the depletion of sensor starts from the junction side and depletion width increases with the increase in the reverse bias, achieving the full depletion of the sensors at $\mathrm{V}_{\mathrm{FD}}$. But for the irradiated sensors, both acceptor and donor traps are created which results in the double junction effect [11]. Hence, for the irradiated sensor depletion starts from both ends of the diode, resulting in the lower full depletion voltage. This is also illustrated in the electric field plots for different bias voltages in figure 4 for fluence $5.10^{14} \mathrm{n}_{\mathrm{eq}} \mathrm{cm}^{-2}$, across the diode bulk. For this fluence, electric field starts growing from both ends of the diode and for reverse bias $\approx 250 \mathrm{~V}$, diode is fully depleted.

From the similar arguments, a qualitative understanding about the different $\mathrm{V}_{\mathrm{FD}}$ trends of neutron and proton irradiated sensors can be achieved. From the trap studies [22, 23], it has been established that in oxygenated silicon (which is the predominantly used nowadays), neutron irradiation introduces mainly acceptor traps along with small amount of donor traps, while charged hadron (protons and pions) irradiation introduces acceptor traps of the similar order but with significant amount of donor traps, particularly E30 trap [23]. E30 trap is a shallow donor trap and contributes additional positive space charge in the proton/pion irradiated sensor bulk. So, on average, the neu- 


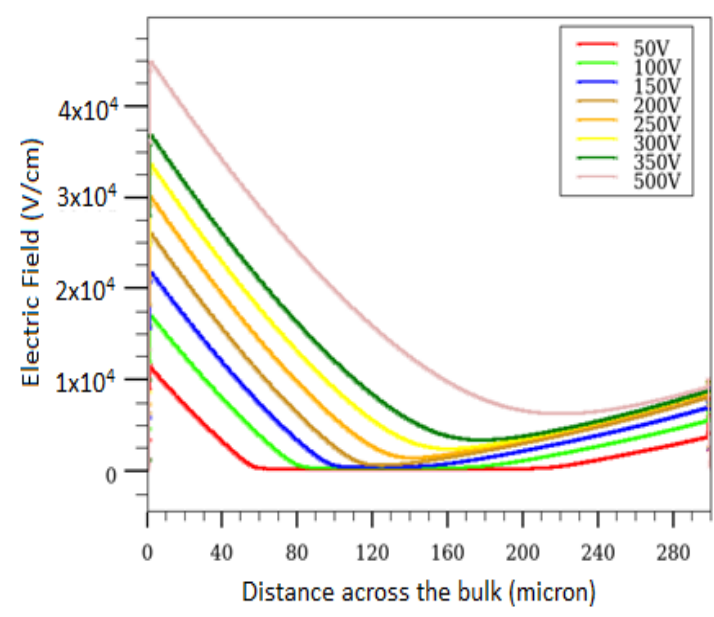

Figure 4: Simulated electric field for different reverse bias voltages across the n-on-p diode bulk, doped with $4.10^{12} \mathrm{~cm}^{-3}$ boron, for proton fluence of $5.10^{14} \mathrm{n}_{\mathrm{eq}} \mathrm{cm}^{-2}$ at $253 \mathrm{~K}$.

tron irradiation results in introduction of mainly negative space charge while proton irradiation results in introduction of both positive and negative space charges. Hence, for p-on-n diode, with initial donor type impurities, the $\mathrm{V}_{\mathrm{FD}}$ lowering happens for neutron as well as for charged hadron irradiation [19]. That effect has been termed as Donor Removal. But, for n-on-p diode, with initial acceptor type impurities, the $\mathrm{V}_{\mathrm{FD}}$ lowering due to double junction takes place for charged hadrons only [20], resulting in apparent Acceptor Removal. This simple interplay of traps and double junction effect can be seen in the Edge-TCT measurements [21] too, where neutron irradiation on p-type bulk results in very high field near $n^{+}$side, while protons and pions irradiation result in much more symmetric electric fields (thus lower $\mathrm{V}_{\mathrm{FD}}$ ).

The observed $\mathrm{V}_{\mathrm{FD}}$ variation with fluence can be easily understood in terms of trap generation without considering the donor/acceptor removal. This approach is not only helpful in understanding the full depletion voltage variation with fluences, it can be useful in understanding the difference of neutron and charged hadron irradiation effects in terms of microscopic traps.

\subsection{Simulations for n-on-p strip sensors}

For n-on-p strip sensors, $\mathrm{R}_{\text {int }}$ is a very important parameter as loss of inter strip isolation leads to spreading of seed signal and results in loss of position resolution. The effect of $\mathrm{Q}_{\mathrm{F}}, \mathrm{N}_{\mathrm{it}}$ and bulk traps is illustrated in figure 5 where simulated $\mathrm{R}_{\text {int }}$ is plotted for $\mathrm{Q}_{\mathrm{F}}$ (without using $\mathrm{N}_{\mathrm{it}}$ and bulk traps), for $\mathrm{Q}_{\mathrm{F}}$ and $\mathrm{N}_{\mathrm{it}}$ together (without bulk traps) and for $\mathrm{Q}_{\mathrm{F}}, \mathrm{N}_{\mathrm{it}}$ and bulk traps taken together (assuming fluence $=1.10^{15} \mathrm{n}_{\mathrm{eq}} \mathrm{cm}^{-2}$ ). While for $\mathrm{Q}_{\mathrm{F}}$ only case, $\mathrm{R}_{\mathrm{int}}$ is very low up to $800 \mathrm{~V}$, addition of interface traps in the simulations improve the $R_{\text {int }}$ quite dramatically. It can be seen that $R_{\text {int }}$ is further improved with the addition of bulk traps. This shows that, along with $\mathrm{Q}_{\mathrm{F}}$ and $\mathrm{N}_{\mathrm{it}}, \mathrm{R}_{\mathrm{int}}$ is affected by bulk damage also and for hadron irradiated sensors the combined simulation approach using surface and bulk damages simultaneously must be used.

The interface traps are acceptor in nature and a significant fraction of them are deep traps. Thus, they can capture free electrons, resulting in compensation of positive oxide charge density. Although bulk damage produces both acceptor and donor type traps, however, during the sensor 

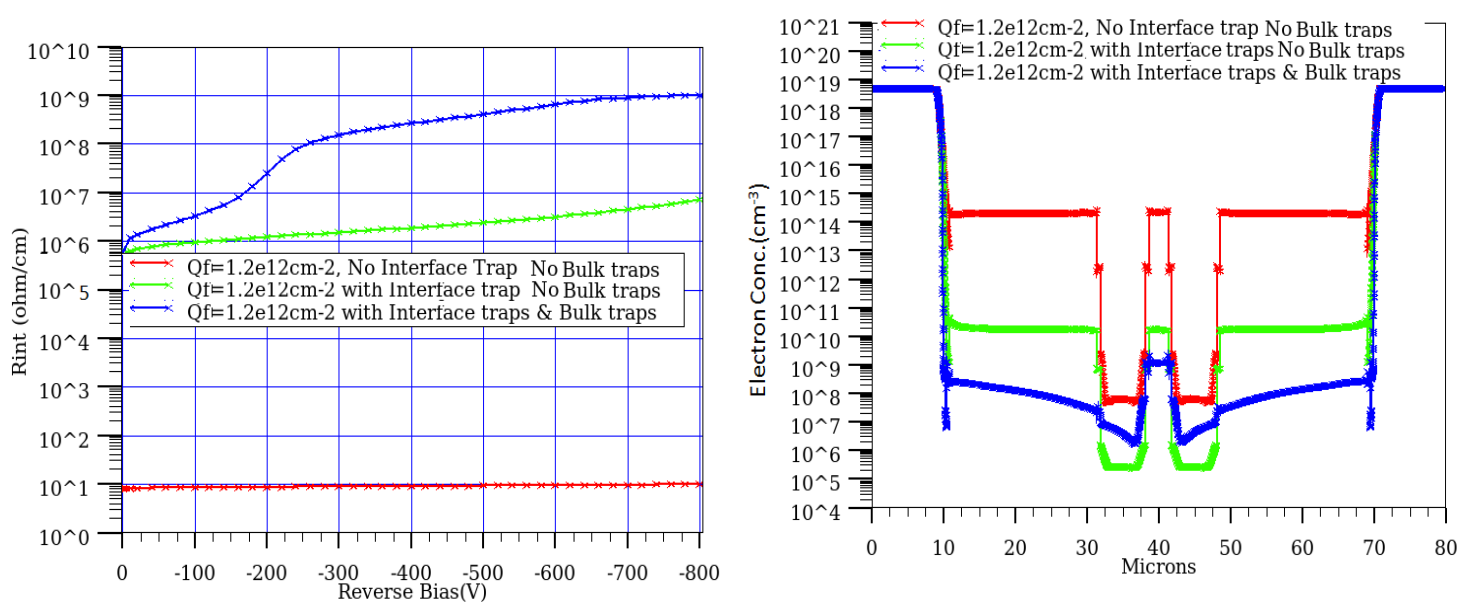

Figure 5: a.) Simulated $R_{\text {int }}$ for $Q_{F}=1.2 \cdot 10^{12} \mathrm{~cm}^{-2}$ with/without interface traps $\left(1.2 .10^{12} \mathrm{~cm}^{-2}\right)$ and bulk traps ( for fluence $=1.10^{15} \mathrm{n}_{\mathrm{eq}} \mathrm{cm}^{-2}$ ), b.) Corresponding electron concentration plots $0.1 \mu \mathrm{m}$ below the $\mathrm{Si}_{-} \mathrm{SiO}_{2}$ interface at $253 \mathrm{~K}$. A double p-stop structure of peak doping density $=5.10^{15} \mathrm{~cm}^{-3}$ is assumed between the $\mathrm{n}^{+}$strips.

operation, mainly acceptor traps are ionized near the $\mathrm{n}^{+}$strips resulting in negative space charge. The negative space charge further reduces the electron concentration and hence improves the $R_{\text {int }}$ values (see figure $5 b$ ).

The simulated $\mathrm{R}_{\mathrm{int}}$ trends for the fluence $1.10^{15} \mathrm{n}_{\mathrm{eq}} \mathrm{cm}^{-2}$ are shown in figure 6 for $\mathrm{n}$-on-p strip sensor with double p-stop isolation structure (6a) and without any isolation structure (6b). Since $\mathrm{Q}_{\mathrm{F}}$ and $\mathrm{N}_{\mathrm{it}}$ are function of several factors [7,8], $\mathrm{R}_{\mathrm{int}}$ simulations are carried out for range of these parameters. The simulated $R_{\text {int }}$ values in figure $6 \mathrm{~b}$ for different amount of surface damage indicate that even apparently low p-stop doing density is capable of maintaining the strip insulation up to proton fluence of $1.10^{15} \mathrm{n}_{\mathrm{eq}} \mathrm{cm}^{-2}$. The $\mathrm{R}_{\mathrm{int}}$ trends for $\mathrm{n}$-on-p strip structure without any $\mathrm{p}$-stop or p-spray isolation are more surprising. For this configuration, $R_{\text {int }}$ values for different amount of surface damages improve with increase in reverse bias and at higher bias voltages, $R_{\text {int }}$ values of the order of $10^{7}$ to $10^{9} \mathrm{Ohm} / \mathrm{cm}$ are possible. The similar observation for n-on-p strip sensor without any isolation structure has been reported in [10].

A comparison of simulated and measured $R_{\text {int }}$ [24] for $n$-on-p strip sensors with double p-stop isolation structure is shown in figure 7. There is significant scattering in the measured values for different samples for a given fluence, so, mean values are plotted in the comparison plot.

\subsection{Radiation hardness comparison for n-on-p and p-on-n strip sensors}

In order to compare the radiation hardness of n-on-p and p-on-n strip sensors, we have studied the electric field distribution within the sensors for different fluence. For irradiated n-on-p strip sensor, maximum electric field is near the $\mathrm{n}^{+}$strip curvature or near the $\mathrm{p}$-stop, just below $\mathrm{Si}_{-} \mathrm{SiO}_{2}$ interface. The former is due to the dense negative space charge created by predominantly acceptor states trapping the electrons moving toward $\mathrm{n}^{+}$strip and it increases with increase the in fluence. Whereas the high electric field near p-stop is due to the electron accumulation layer formed due to $\mathrm{Q}_{\mathrm{F}}$ and increases with increase in $\mathrm{Q}_{\mathrm{F}}$. The electric field near $\mathrm{p}$-stop is also a strong function of 

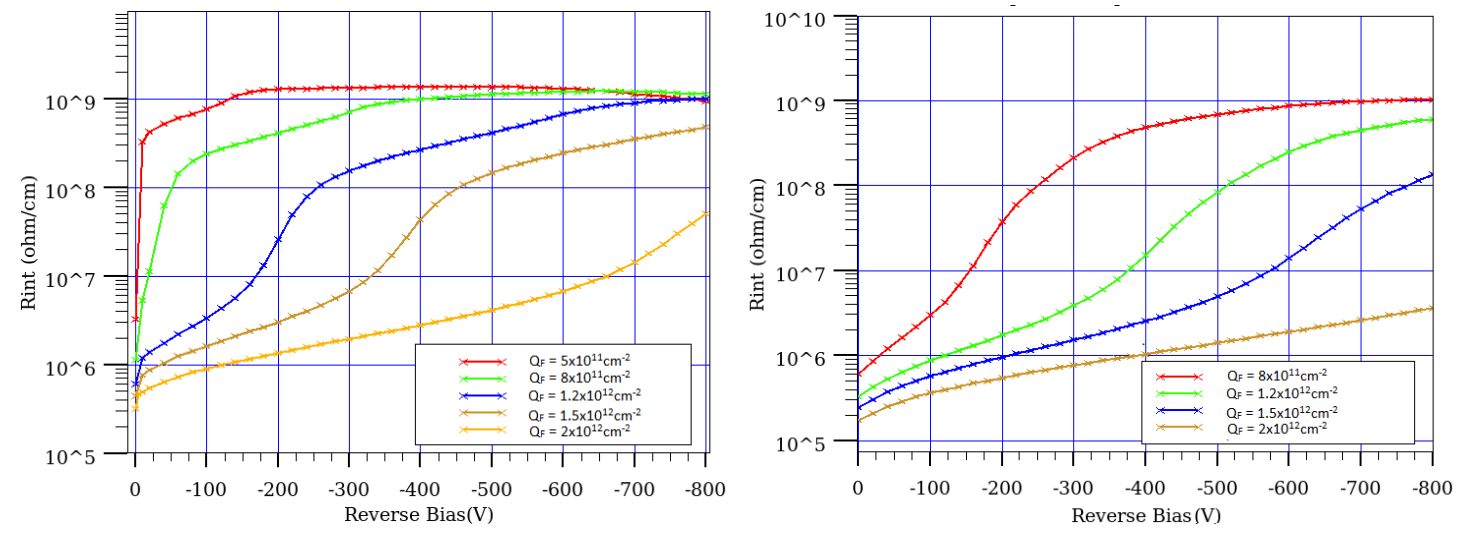

Figure 6: a.) Simulated $R_{\text {int }}$ vs reverse bias for fluence $=1.10^{15} \mathrm{n}_{\mathrm{eq}} \mathrm{cm}^{-2}$ with double p-stop isolation structure (p-stop doping density $=5.10^{15} \mathrm{~cm}^{-3}$ ) and $\mathrm{b}$.) without any isolating structure for different values of $\mathrm{Q}_{\mathrm{F}}$ and $\mathrm{N}_{\mathrm{it}}$. The $\mathrm{N}_{\mathrm{it}}$ density is assumed to be equal to $\mathrm{Q}_{\mathrm{F}}$ density.

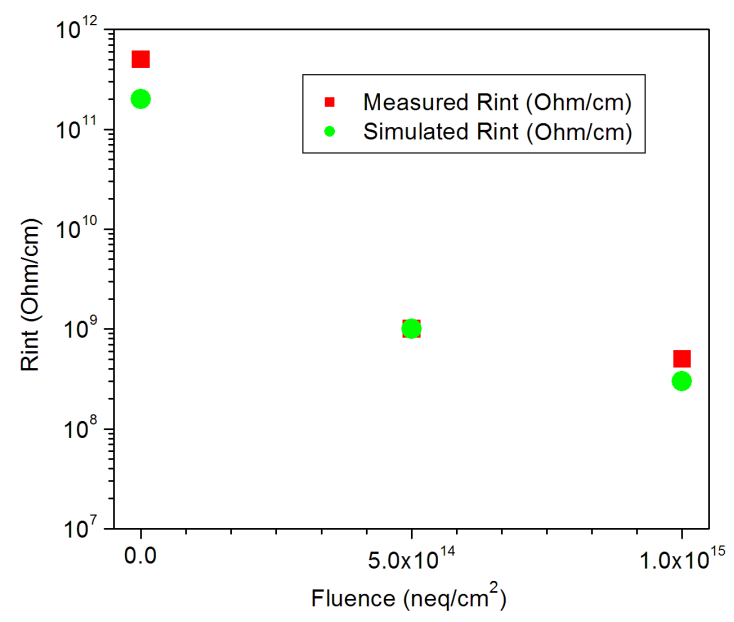

Figure 7: Comparison of Simulated and measured $\mathrm{R}_{\text {int }}$ for different fluence for $\mathrm{n}$-on-p strip sensors with double p-stop at $600 \mathrm{~V}$. The $\mathrm{R}_{\mathrm{int}}$ simulations for fluences $\left(\mathrm{n}_{\mathrm{eq}} \mathrm{cm}^{-2}\right) 0,5 \cdot 10^{14}, 1.10^{15}$ are carried out using $\mathrm{Q}_{\mathrm{F}}$ (which is equal to $\mathrm{N}_{\mathrm{it}}$ ) values of $1.10^{11} \mathrm{~cm}^{-2}, 1.10^{12} \mathrm{~cm}^{-2}$ and $1.5 .10^{12} \mathrm{~cm}^{-2}$ respectively.

p-stop doping densities and it is suggested to avoid very high p-stop doping densities [15].

For irradiated p-on-n strip sensor, maximum electric field is near the $\mathrm{p}^{+}$strip, just below the Si$\mathrm{SiO}_{2}$ interface where the electron accumulation layer due to $\mathrm{Q}_{\mathrm{F}}$ comes in contact with $\mathrm{p}^{+}$strip doping. Simulated electric field contours near the strips for n-on-p and p-on-n strip sensors are shown in figures $8 \mathrm{a}$ and $8 \mathrm{~b}$ respectively.

The comparison of maximum electric fields for irradiated $n-o n-p$ and $p-o n-n$ strip sensors is very important for the appropriate sensor polarity selection. Electric fields for $n-o n-p$ and $p$-on-n strip sensors, irradiated with proton fluence $1.10^{15} \mathrm{n}_{\mathrm{eq}} \mathrm{cm}^{-2}$, are compared in figure 9 at the distance of $0.1 \mu \mathrm{m}$ below $\mathrm{Si}_{-} \mathrm{SiO}_{2}$ (where p-on-n strip sensor have maximum field) and for the distance of $1.4 \mu \mathrm{m}$ below $\mathrm{Si}_{-} \mathrm{SiO}_{2}$ (where n-on-p strip sensor have maximum field). In these simulations surface damage is implemented assuming $\mathrm{Q}_{\mathrm{F}}$ and $\mathrm{N}_{\mathrm{it}}$ values equal to $1.2 .10^{12} \mathrm{~cm}^{-2}$ along with the bulk damage for fluence of $1.10^{15} \mathrm{n}_{\mathrm{eq}} \mathrm{cm}^{-2}$. From electric field comparison, it is clear that 

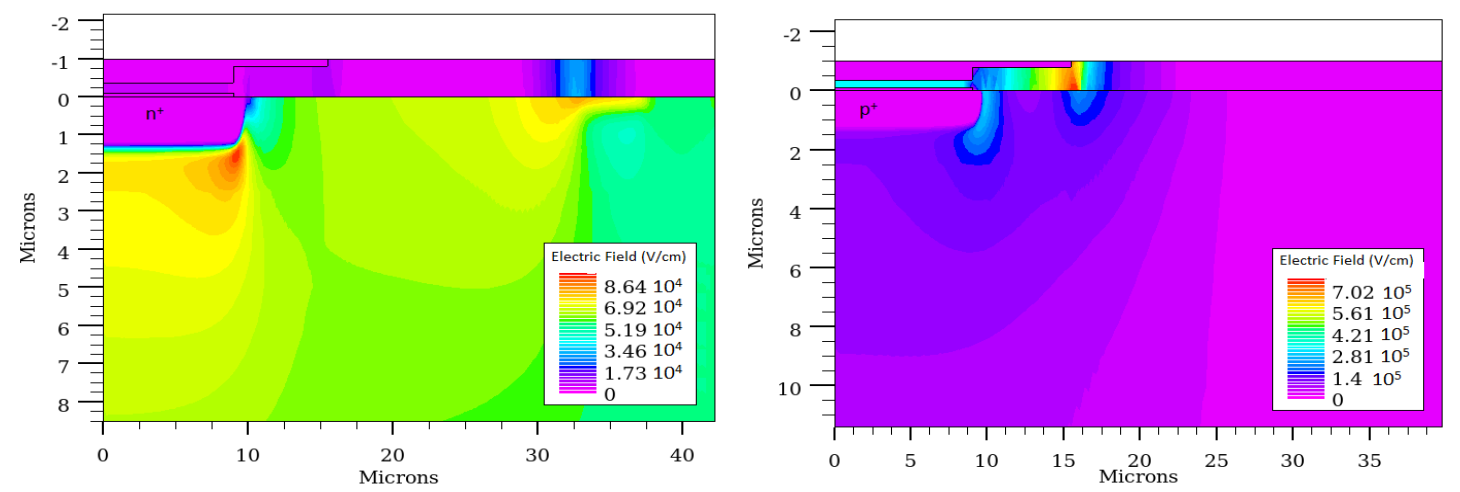

Figure 8: a.) Electric field contours near the strips of n-on-p strip sensors and for b.) p-on-n strip sensors for fluence $1.10^{15} \mathrm{n}_{\mathrm{eq}} \mathrm{cm}^{-2}$ for reverse bias $500 \mathrm{~V}$

very high electric fields get developed near $\mathrm{p}^{+}$strips, due to surface damage, making $\mathrm{p}$-on-n strip sensors more prone to breakdown [24]. These results also highlight the importance of simultaneous incorporation of bulk and surface damages in the simulations. Further, for p-on-n strip sensors, increase of surface damage results in higher electric field near $\mathrm{p}^{+}$strips, while the higher surface damage in $n-o n-p$ strip sensor results in lowering of maximum electric field near $\mathrm{n}^{+}$curvature.
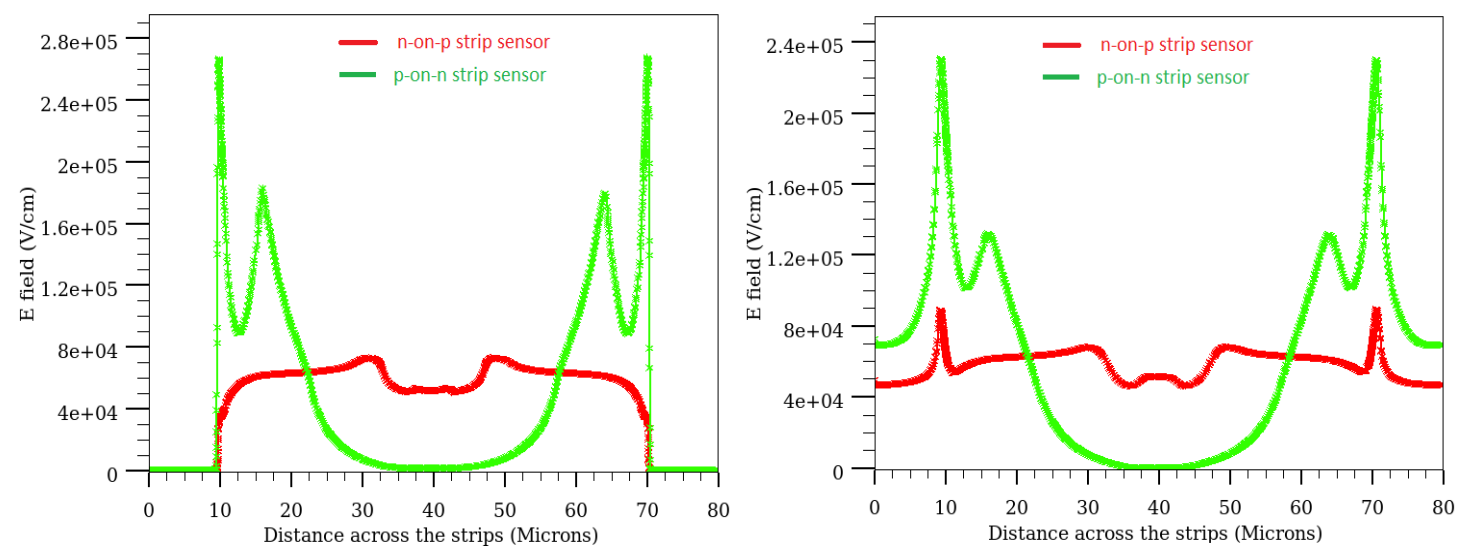

Figure 9: a.) Comparison of electric field for $n-o n-p$ and $p-o n-n$ strip sensors for the cut line $0.1 \mu \mathrm{m}$ below the $\mathrm{Si}-\mathrm{SiO}_{2}$ and for b.) cut line $1.4 \mu \mathrm{m}$ below the $\mathrm{Si}-\mathrm{SiO}_{2}$ interface for reverse bias $500 \mathrm{~V}$ for fluence $1.10^{15}$ $\mathrm{n}_{\mathrm{eq}} \mathrm{cm}^{-2}$

\section{Summary}

Radiation damage simulation for the silicon sensors can be very helpful in understanding the degrading effects of irradiation and for the sensor parameters optimization for the future upgrades of the LHC. Since for the silicon strip and pixel sensors used in hadron environment, both the bulk and surface damages take place simultaneously, it becomes essential to implement both of these degrading effects in the simulations for reliable predictions. Simulations carried out using either bulk or surface damage alone can be misleading. Moreover, implementation of surface damage 
must be carried out incorporating both oxide charges and interface traps. The interface traps can play vital role in determining the interface properties due to high density of deep interface traps. In this work, we have used an effective two trap model to implement the bulk damage due to proton irradiation. The surface damage implementation is carried out using the measured oxide charge densities and interface traps. For simplification, we have assumed that the interface trap states can be represented by one deep acceptor state and one shallow acceptor state.

The $\mathrm{V}_{\mathrm{FD}}$ simulations indicate that the $\mathrm{V}_{\mathrm{FD}}$ variation with fluence can be understood in terms of traps rather than as an outcome of donor/acceptor removal. The interpretation of $\mathrm{V}_{\mathrm{FD}}$ in terms of traps may be helpful in understanding the higher $\mathrm{V}_{\mathrm{FD}}$ values for the neutron irradiated sensors as compared to that of the proton one. $\mathrm{R}_{\text {int }}$ simulations indicate that good interface resistance is expected for the n-on-p strip sensors having low doping p-stops isolation structure or even without any isolation structure irradiated with very high fluence of protons. The electric field simulations for $n-o n-p$ and $p$-on-n strip sensors indicate that n-on-p sensors are more suitable for the high fluence application.

\section{Acknowledgment}

Authors would like to thank Indo-Swiss Joint Research Program (ISJRP), Department of Science and Technology (DST) and University of Delhi for providing financial assistance for this research. The work was partly performed in the framework of the RD50 collaboration. We would like to thank Michael Moll for many helpful discussion and suggestions. Ranjeet and Geetika would like to thank UGC and CSIR (India) for providing the research grant.

\section{References}

[1] F. Hartmann, Silicon tracking detectors in high-energy physics, NIM A 666, 25 - 46 (2012).

[2] C. P. Nuttens, Overview of the CMS Strip and Pixel Detectors, PoS (Vertex 2013) 002.

[3] The ATLAS Collaboration, Operation and Performance of the ATLAS Semiconductor Tracker, JINST 9, P08009 (2014).

[4] A. Dierlamm, Silicon Sensor Developments for the CMS Tracker Upgrade, JINST 7, C01110 (2012).

[5] S. Kuehn, Atlas Phase - 2 Strip Tracker Upgrade, PoS (VERTEX 2013) 013.

[6] The RD50 collaboration; http://cern.ch/rd50.

[7] T. Pohlsen, Charge Losses in Silicon Sensors and Electric Field Studies at the $\mathrm{Si}_{-} \mathrm{SiO}_{2}$ Interface, $\mathrm{PhD}$ thesis, University of Hamburg, 2013.

[8] J. Zhang, X-ray Radiation Damage Studies and Design of a Silicon Pixel Sensor for Science at the XFEL, PhD thesis, University of Hamburg, 2013.

[9] C. Piemonte, Device Simulations of Isolation Techniques for Silicon Microstrip Detectors Made on p-type Substrates, IEEE Trans. Nucl. Sci. NS-53 (3) (2006) 1694.

[10] Y. Unno et al., p-Bulk sSlicon Microstrip Sensors and Irradiation, NIN A 579 (2007) 614.

[11] V. Eremin et al., The Origin of Double Peak Electric Field Distribution in Heavily Irradiated Silicon Detectors, NIM A 476 (2002) 556. 
[12] M. Petasecca et al., Numerical Simulation of Radiation Damage Effects in p-Type and n-Type FZ Silicon Detectors, IEEE Trans. Nucl. Sci. NS-53(5) (2006) 2971.

[13] M. Swartz et al., Observation, Modeling, and Temperature Dependence of Doubly Peaked Electric Fields in Irradiated Silicon Pixel Sensors, NIM A 565 (2006) 212.

[14] ATLAS Silvaco Version 5.15.32.R Nov 2009, Users Manual, http://www.silvaco.com

[15] R. Dalal et al., Combined Effect of Bulk and Surface Damage on Strip Insulation Properties of Proton Irradiated $n^{+}$-p Silicon Strip Sensors, JINST 9, P04007 (2014).

[16] R. Dalal et al., TCAD Simulation of Irradiated Silicon Sensors, presented at 25th RD50 Workshop, CERN, Nov. 2014.

[17] M. Moll, Radiation Damage in Silicon Particle Detectors, dissertation, DESY-THESIS-1999-040, University of Hamburg, Hamburg Germany (1999).

[18] M. Murat et al.,Spatial Distribution of Electron-Hole Pairs Induced by Electrons and Protons in $\mathrm{SiO}_{2}$, IEEE Trans. Nucl. Sci., 51 (2004) 3211âĂŞ3218.

[19] G. Lindstorm et al., Radiation Hard Silicon Detectors - Developments by the RD48 (ROSE) Collaboration, NIM A 466, 308 - 326 (2001).

[20] G. Kramberger et al., Initial Acceptor Removal in p-type silicon Detectors, presented at 23rd RD50 Workshop, CERN, Nov. 2013.

[21] G. Kramberger et al., Modeling of Electric Field in Silicon Micro-strip Detectors Irradiated with Neutrons and Pions, JINST 9, P00016 (2014).

[22] R. Radu et al., Investigation of Point and Extended Defects in Electron Irradiated Silicon Dependence on the Particle Energy, presented at 24th RD50 Workshop, Bucharest, June 2014.

[23] I. Pintilie et al., Radiation Induced Point and Cluster Related Defects with Strong Impact on Damage Properties of Silicon Detectors, NIM A 611, 52 - 68 (2009).

[24] A. Dierlamm, Planar Sensors for Future Vertex and Tracking Detectors, PoS (VERTEX 2013) 027 